\section{(6) OPEN ACCESS}

\title{
POU2F2-oriented network promotes human gastric cancer metastasis
}

\author{
Si-Meng Wang, ${ }^{1}$ Jun Tie, ${ }^{1}$ Wen-Lan Wang, ${ }^{2}$ Si-Jun Hu, ${ }^{1}$ Ji-Peng Yin, ${ }^{1}$ Xiao-Fang Yi, ${ }^{1}$ \\ Zu-Hong Tian, ${ }^{1}$ Xiang-Yuan Zhang, ${ }^{1}$ Meng-Bin Li, ${ }^{1}$ Zeng-Shan Li, ${ }^{3}$ Yong-Zhan Nie, ${ }^{1}$ \\ Kai-Chun Wu, ${ }^{1}$ Dai-Ming Fan ${ }^{1}$
}

\begin{abstract}
- Additional material is published online only. To view please visit the journal online (http://dx.doi.org/10.1136/ gutjnl-2014-308932).

${ }^{1}$ State Key Laboratory of Cancer Biology and Xijing Hospital of Digestive Diseases, Xijing Hospital, Fourth Military Medical University, Xi'an, Shaanxi, China

${ }^{2}$ Department of Aerospace Hygiene and Health Service, School of Aerospace Medicine, Fourth Military Medical University, Xi'an, Shaanxi, China

${ }^{3}$ Department of Pathology, Xijing Hospital, Fourth Military Medical University, Xi'an, Shaanxi, China
\end{abstract}

\section{Correspondence to} Dr. Jun Tie or Professor Daiming Fan, State key Laboratory of Cancer Biology and Xijing Hospital of Digestive Diseases, Xijing Hospital, the Fourth Military Medical University, No. 127, West Chang-Le Road, Xi'an 710032, China;

tiejun7776@163.com or fandaim@fmmu.edu.cn

Received 1 December 2014 Revised 8 April 2015 Accepted 28 April 2015 Published Online First 27 May 2015

\begin{abstract}
Background and aims Aberrant upregulation of POU2F2 expression has been discovered in metastatic gastric cancer (GC). However, the mechanisms underlying the aberrant upregulation and the potential functions of POU2F2 remain uncertain.

Design The role and mechanism of POU2F2 in GC metastasis were investigated in gastric epithelial cells, GC cell lines and an experimental metastasis animal model by gain of function and loss of function. Upstream and downstream targets of POU2F2 were selected by bioinformatics and identified by luciferase reporter assay, electrophoretic mobility shift assay and chromatin immunoprecipitation PCR. The influence of miR-218 on its putative target genes (POU2F2, ROBO1 and IKK- $\beta$ ) and GC metastasis was further explored via in vitro and in vivo approaches.
\end{abstract}

Results Increased POU2F2 expression was detected in metastatic GC cell lines and patient samples. POU2F2 was induced by the activation of nuclear factor (NF)- $\kappa B$ and, in turn, regulated ROBO1 transcription, thus functionally contributing to GC metastasis. Finally, miR-218 was found to suppress GC metastasis by simultaneously mediating multiple molecules in the POU2F2-oriented network.

Conclusions This study demonstrated that NF- $\kappa B$ and the SLIT2/ROBO1 interaction network with POU2F2 as the central part may exert critical effects on tumour metastasis. Blocking the activation of the POU2F2oriented metastasis network using miR-218 precursors exemplified a promising approach that sheds light on new strategies for GC treatment.

\section{INTRODUCTION}

POU2F2, a member of the POU transcription factor family, is a B-cell-specific octamer transcription factor. ${ }^{1}$ Previous studies have indicated that POU2F2 is normally expressed in B cells and B cell lineage tumour cells ${ }^{2-4}$ to regulate Ig, B cell proliferation and $\mathrm{B}$ cell differentiation genes. ${ }^{5}$ Recent studies have identified POU2F2 expression in pancreatic cancer, ${ }^{6}$ gastric cancer $(\mathrm{GC})^{7}$ and other epithelial tumours. However, the potential functions of POU2F2 and the exact mechanisms governing POU2F2 expression in GC are poorly understood.

In this study, POU2F2 expression was detected in both GC cells with high metastatic potential and GC tissues with lymph node or distant metastasis. GC patients with positive POU2F2 expression had

\section{Significance of this study}

What is already known on this subject?

- Upregulation of POU2F2 has been shown to be associated with gastric cancer (GC) metastasis.

- Overexpression of nuclear factor (NF)- $\kappa \mathrm{B}$ and POU2F2 is found in cultured Hodgkin/ReedSternberg cells.

- miR-218 expression is decreased in GC, and the restoration of miR-218 suppresses tumour cell invasion and metastasis.

\section{What are the new findings?}

- POU2F2 promoted GC metastasis by a positive regulation of $\mathrm{ROBO1}$. The overall survival in $\mathrm{GC}$ patients with positive POU2F2 was remarkably reduced.

- Upregulation of POU2F2 in metastatic GC was achieved not only through the transcriptional activation by NF- $\mathrm{KB}$ but also by eliminating the repression of miR-218 at the post-transcriptional level.

- The interaction between NF- $\kappa B$ and the SLIT2I ROBO1 pathway linked by POU2F2 contributed to gastric cancer metastasis.

- miR-218 impeded metastasis by orchestrating multiple targets of the POU2F2-oriented network.

How might it impact on clinical practice in the foreseeable future?

- The discovery of this miR-218-NF-KB/POU2F2/ SLIT2/ROBO1 axis of a signal transduction pathway will aid in a better understanding of the pathogenic mechanisms of GC metastasis. Inhibiting NF-KB/POU2F2/SLIT2/ROBO1 signal transduction with miR-218 indicates a feasible and promising approach that may be applicable to the treatment of GC.

a shorter survival compared with patients with negative POU2F2 expression. Moreover, POU2F2 downregulation significantly hampered the metastatic ability of GC cells. In contrast, POU2F2 upregulation in low metastatic potential cells advocated their invasion and metastasis. Further studies indicated that POU2F2 directly triggered ROBO1 transcription. ROBO1, a single-channel transmembrane receptor, can promote cancer metastasis and 
endothelial cell migration through interacting with ligands of the SLIT family. ${ }^{8-10}$ In mammals, three SLIT proteins (SLIT1-3) have been discovered. ${ }^{11}$ However, SLIT1 expression is specific to the brain, ${ }^{12}$ and SLIT3 expression is significantly reduced in GC tissue compared with normal gastric tissue. ${ }^{8}$ Thus, we deduced and confirmed that POU2F2 induces metastatic potential through the SLIT2/ROBO1 pathway.

Previous studies have found that POU2F2 expression closely correlates with nuclear factor (NF)- $\kappa \mathrm{B}$ status in Hodgkin/ReedSternberg cells ${ }^{13}$ and precursor B lymphocytes, ${ }^{14}$ thereby suggesting that NF- $\mathrm{KB}$ may regulate POU2F2 expression. However, no evidence has directly supported specific regulation of POU2F2 by NF-кB. In the present study, the relationship between NF- $\kappa \mathrm{B}$ activity and POU2F2 expression was thoroughly investigated for the first time. The bioinformatic results, promoter reporter gene assay, chromatin immunoprecipitation (ChIP) assay and electrophoretic mobility shift assay (EMSA) demonstrated that NF- $\mathrm{BB}$ positively regulated POU2F2 expression by directly binding to the POU2F2 promoter region $(-531$ to -522 ; TGATCTTCCC). These results illustrated the possible causes and regulatory machinery of ectopic POU2F2 expression in GC.

microRNAs (miRNAs) play a critical role in tumour occurrence and development through negatively regulating target gene expression. ${ }^{15} 16$ Although POU2F2 is regulated at the transcriptional level by NF- $\mathrm{B}$, it is unknown whether POU2F2 is also governed by miRNAs at the post-transcriptional level. Bioinformatics analysis indicated that miR-218 might regulate POU2F2 expression by binding its 3 -untranslated region (UTR). In recent years, a number of studies have determined that miR-218 functions as a tumour-suppressive miRNA. ${ }^{17-22}$ In particular, our previous results demonstrated that miR-218 inhibits invasion and metastasis of GC by targeting the ROBO1 receptor, ${ }^{8}$ which was further validated in various cell models. $^{21}{ }^{23-26}$ miR-218 may also suppress the activity of the $\mathrm{NF}-\kappa \mathrm{B}$ signalling pathway by directly inhibiting the expression of IKK- $\beta^{27}$ and $\mathrm{ECOP}^{28}$ in GC cells, thus suggesting that miR-218 may simultaneously repress the NF- $\mathrm{BB}$ and SLIT2/ ROBO1 signalling pathways. This study further demonstrated that miR-218 directly and negatively regulates POU2F2 together with IKK- $\beta$ and ROBO1. miR-218 overexpression simultaneously inhibited the expression of IKK- $\beta$, POU2F 2 and ROBO1, and suppressed the NF- $\mathrm{KB}$ and SLIT2/ROBO1 signalling pathways, thereby preventing GC cell metastasis.

This study showed, for the first time, the effects and mechanisms of POU2F2 in promoting GC cell metastasis, and it also revealed that the POU2F2-centric interaction networks (the NF-кB/POU2F2/SLIT2/ROBO1 network) might play an essential role in GC metastasis. The tumour suppressor miR-218 may act simultaneously on POU2F2 and the NF- $\mathrm{KB}$ activation factor, IKK- $\beta$ kinase, in its upstream signalling pathway as well as its downstream target gene, ROBO1, to impede GC metastasis.

\section{MATERIALS AND METHODS \\ Clinical samples}

The following two types of GC tissue microarrays were purchased from Shanghai Outdo Biotech: HStm-Ade180Sur-02, which contains 90 cases of gastric adenocarcinoma and paired paraneoplastic tissues with one point for each tissue; and HStm-Ade178Sur-01, which contains 67 cases of matched cancer/para-cancer samples, 39 cases of unpaired cancer tissues and 5 cases of single para-cancer tissues with one for point each tissue. The Tissue Microarray XJ-CT-Gas01 was generated with 70 cases of matched cancer and para-cancer tissues legally collected in our centre with one point for each tissue. All patients were followed up from 1 to 120 months after the operation, and all specimens involved were determined by $\mathrm{H} \& \mathrm{E}$ staining.

\section{Western blot analysis}

Western blot analysis was performed using standard procedures. The antibodies used in the western blot analysis are listed in the online supplementary experimental procedures.

\section{ChIP-PCR analysis}

The ChIP-PCR assay was conducted as previously described ${ }^{29}$ with minor modifications. The detailed procedures are described in the online supplementary experimental procedures.

\section{Electrophoretic mobility shift assay}

The procedures are detailed in the online supplementary experimental procedures.

\section{Statistical analyses}

All statistical analyses were conducted using the SPSS software statistical package (V.17.0). The differences between the two groups were assessed using Student's t test. Overall survival curves were estimated by the Kaplan-Meier method, and the difference in survival was evaluated using the log-rank test. p Values $<0.05$ were considered statistically significant.

\section{RESULTS \\ POU2F2 expression is increased in GC cells with high metastatic potential and metastatic GC tissues}

POU2F2 expression was analysed in a variety of cell lines, which included normal gastric epithelial cells (GES-1), cell sublines with strong (SGC7901-M and MKN28-M) or weak (MKN28-NM and SGC7901-NM) metastatic ability derived from GC cell lines (SGC7901-M and MKN28-M). The results showed that compared with GES-1 cells POU2F2 mRNA expression increased by 1.4 -fold in MKN28-NM and SGC7901-NM cells. However, in the highly metastatic GC cell lines SGC7901-M and MKN28-M, the expression was increased by approximately 12-16 times (see online supplementary figure S1). High levels of POU2F2 protein were detected in SGC7901-M and MKN28-M cells, but extremely low levels of POU2F2 protein were detected in GES-1, MKN28-NM and SGC7901-NM cells (figure 1A).

Furthermore, POU2F2 expression was examined in 266 cases of clear metastatic state of GC and 232 cases of cancer adjacent tissues. Compared with para-cancerous tissues, no POU2F2 expression differences were observed in GC tissues without metastasis, while there was an obvious increase of POU2F2 expression in metastatic GC tissues (figure 1B). According to the expression of POU2F2 in GC tissues, 90 cases of GC (HStm-Ade180Sur-02 tissue array) were divided into those with POU2F2-positive expression $(n=65)$ and those with POU2F2-negative expression $(n=25)$. The relationship of POU2F2 with overall survival was analysed, and the overall survival in patients with positive POU2F2 expression was remarkably reduced (figure 1C). The multivariate analysis results suggested that the POU2F2 protein expression status might have a potential to be an independent prognostic indicator for GC ( $p=0.0023, \mathrm{HR}=167,95 \% \mathrm{CI} 0.036$ to 0.780 ; see online supplementary tables S1 and S2). The results implied that POU2F2 upregulation might give rise to $\mathrm{GC}$ metastasis. 
A

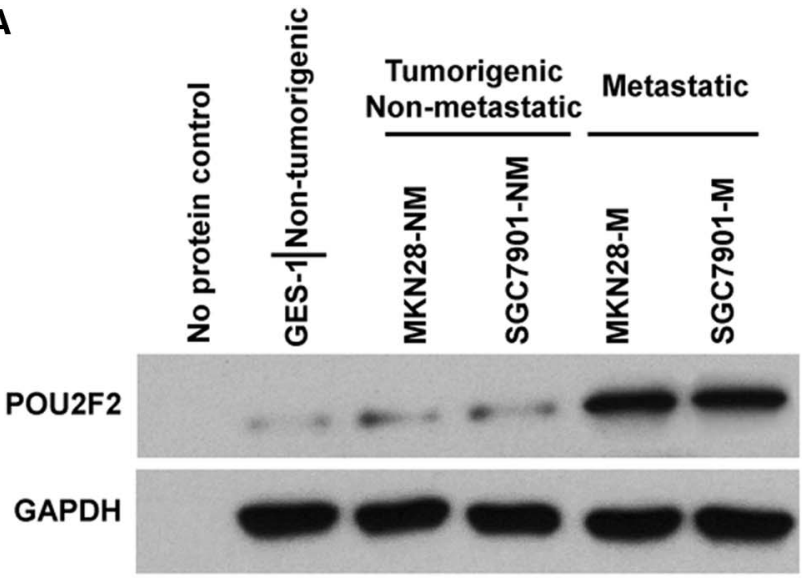

B

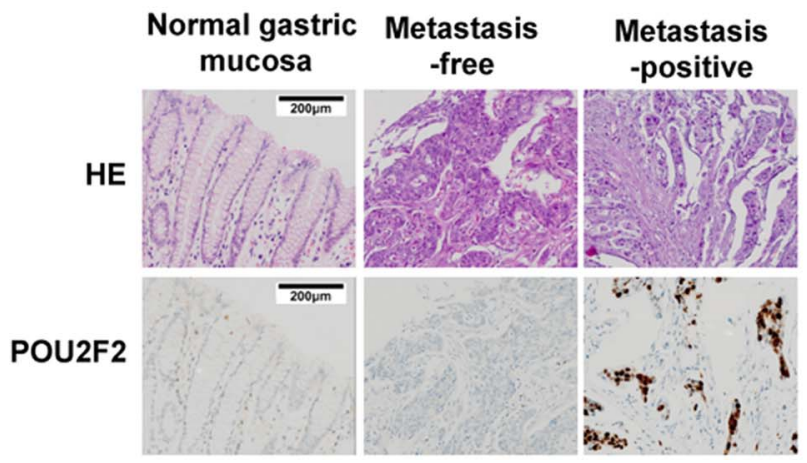

C
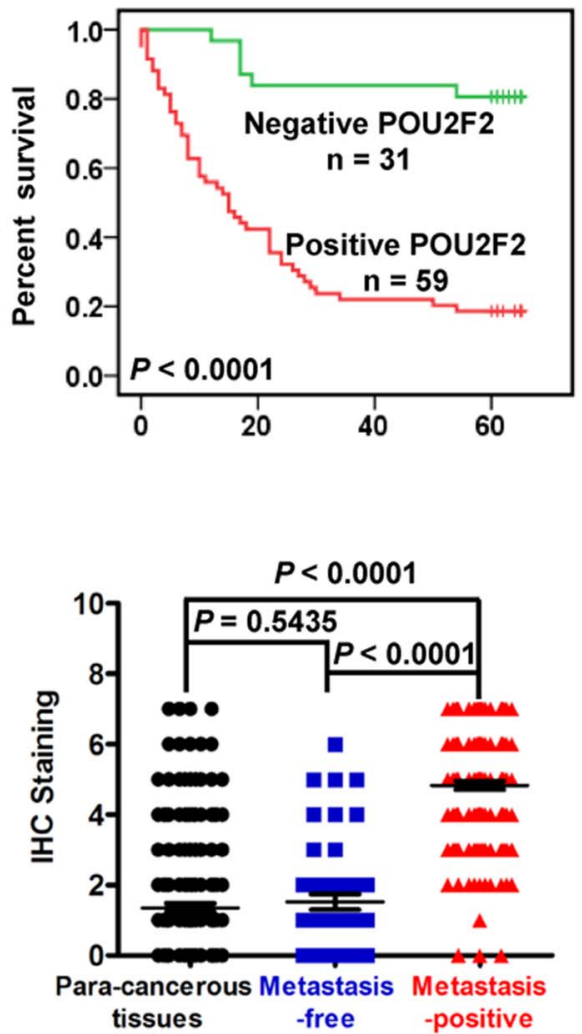

Figure 1 POU2F2 levels correlate positively with metastatic ability of gastric cancer (GC). (A) Western blot for POU2F2 in five human gastric cell lines. Glyceraldehyde 3-phosphate dehydrogenase (GAPDH) was a loading control, and $\mathrm{H}_{2} \mathrm{O}$ is a no protein control $(n=3)$. (B) Left:

immunohistochemical staining for POU2F2 in primary GCs from patients with the indicated status of metastasis and normal tissues. Right: POU2F2 expression levels were scored with semiquantitative immunohistochemical $(I H C)$ analysis. POU2F2 was upregulated in metastatic GC $(n=200)$, while it was scarcely detected in normal gastric mucosa $(n=232)$ and $G C$ tissues without metastasis $(n=66)$. (C) Kaplan-Meier curves depicting overall survival for patients with $\mathrm{GCs}(\mathrm{n}=90)$. The curves were stratified based on POU2F2 expression. Overall survival was defined as the interval between the date of the surgery and the date of death or last follow-up.

\section{POU2F2 positively regulates GC invasion and metastasis in vitro and in vivo}

We adopted loss-of-function and gain-of-function experiments to study POU2F2 function in GC metastasis. First, four POU2F2-specific siRNAs were transfected into SGC7901-M cells. Based on the western blot analysis, the siRNA2 with the highest inhibition rate was selected to construct the shPOU2F2 lentiviral vector for further functional experiments (see online supplementary figure S2). The results found that compared with control cells SGC7901-M and MKN28-M cells transfected with shPOU2F2 had significantly reduced POU2F2 levels (figure $2 \mathrm{~A}$ ), and the in vitro invasive ability was correspondingly decreased by $2-3$ times (figure $2 \mathrm{~B}$ ). In nude mice with intravenous injection of visceral metastasis, the lung metastases resulting from the injection of SGC7901-M cells infected with shPOU2F2 were significantly reduced compared with controls (figure 2C). In addition, we used the POU2F2 lentiviral vector to upregulate POU2F2 expression in the non-metastatic GC SGC7901-NM cells and in the normal gastric mucosa GES-1 cells (figure 2A), resulting in a robust increase in the invasion ability of infected cells (figure 2B). Meanwhile, the lung metastases in nude mice injected with SGC7901-NM-POU2F2 cells significantly increased (figure 2C). We constructed a shRNA-resistant POU2F2 lentiviral vector, POU2F2 $\Delta$, via sitedirected mutagenesis. In SGC7901-M-shPOU2F2 cells, the recovery of POU2F2 expression with this shRNA-resistant vector restored the high metastatic ability of the cells (figure 2D). In summary, the above results illustrated that POU2F2 may be critical for the invasion and metastasis of GC cells.

POU2F2 may affect GC metastasis by the positive regulation of ROBO1

To uncover the mechanism of POU2F2 in GC, the target genes that may be regulated by POU2F2 were analysed using TRANSFAC (http://www.gene-regulation.com/index2. $\mathrm{html})$. Bioinformatics analysis revealed that the promoter region of the ROBO1 gene ( -880 to -873$)$ contained a POU2F2binding sequence $\left(5^{\prime} \text {-ATTTGCAT- } 3^{\prime}\right)^{30}$ (figure $3 \mathrm{~A}$ ), thus indicating that ROBO1 may be a target gene of POU2F2. To confirm this hypothesis, ChIP-PCR and sequencing results verified that POU2F2 binds to the ROBO1 promoter (figure 3B, see online supplementary figure $\mathrm{S} 3$ ). The POU2F2 expression vector was co-transfected with the ROBO1 full-length promoter reporter gene or the binding-site mutant reporter gene into SGC7901-NM, HeLa and 293T cells (three cell lines with low POU2F2 expression). In the above three cell lines, POU2F2 regulated the transcriptional activity of ROBO1 wild-type promoters. In the presence of the -880 to -873 binding site mutation, the regulatory effect disappeared, thereby suggesting that POU2F2 
A

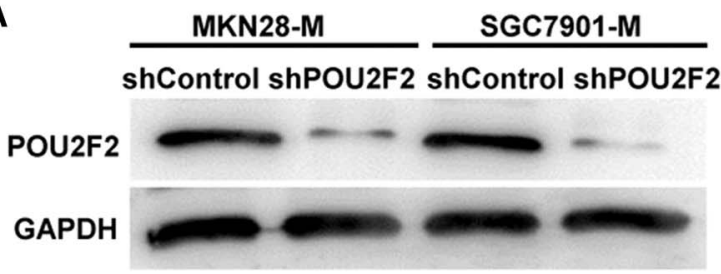

B

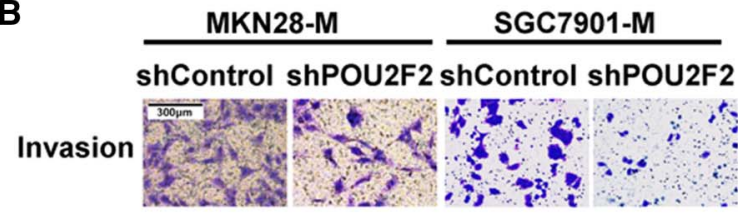

$P=0.0023$

$P=0.0010$

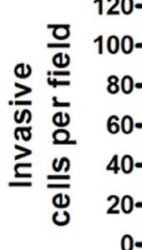

0.002
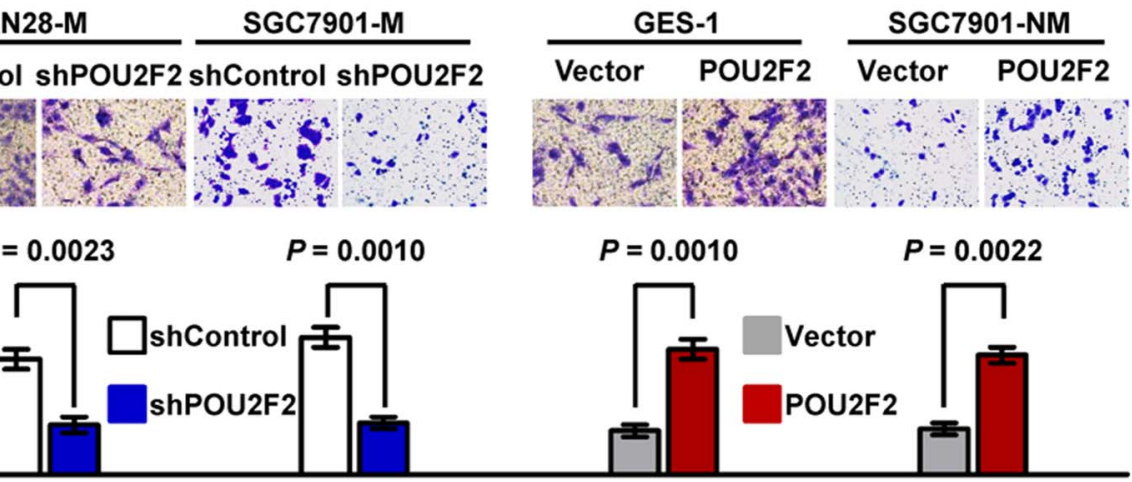

$P=0.0010$

$P=0.0022$
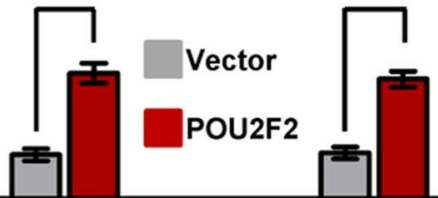

C
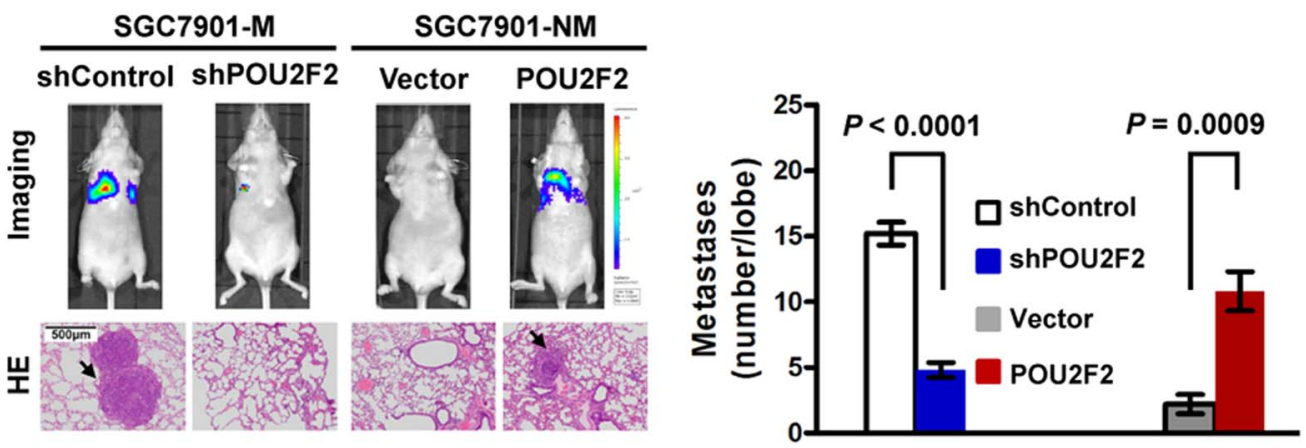

D
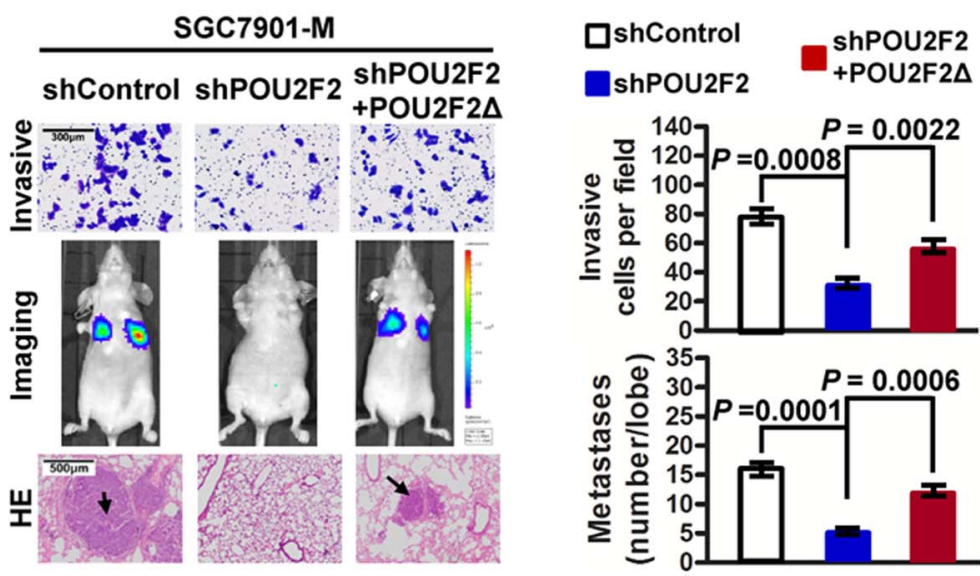

Figure 2 POU2F2 positively regulates metastasis in gastric cancer (GC). (A) Western blot for POU2F2 in SGC7901-M and MKN28-M cells infected with shPOU2F2 or control shRNA lentiviral vector as well as in SGC7901-NM and gastric epithelial cells (GES)-1 infected with the POU2F2-expressing or empty control vector $(n=3)$. (B) Invasion assays after transfection of shPOU2F2 or POU2F2-expressing vectors in the indicated cells $(n=3)$. POU2F2 enhanced GC cell invasion. (C) SGC7901-M and SGC7901-NM cells expressing a luciferase reporter were transfected with the indicated constructs and were injected into the nude mice via the tail vein. After 6 weeks, bioluminescence imaging (BLI) showed that there were significantly more metastasis lesions in the lung of SGC7901-M-control and SGC7901-NM-POU2F2 mice than in the corresponding control groups. The results of H\&E staining were the same as those of BLI ( $n=5)$. (D) Western blot for POU2F2 in the indicated SGC7901-M cells transfected with shPOU2F2 or the shRNA-resistant expression construct, POU2F2 $\Delta$. POU2F2 expression was recovered in the SGC7901-M-shPOU2F2 cells transfected with POU2F2 $\Delta$ (left). POU2F2 $\Delta$ restored the high metastatic ability of the cells. The representative Transwell images, BLI and H\&E-stained sections of the lung tissues collected from indicated groups 6 weeks after tail vein injection are shown in the middle panel. Quantitative analysis was performed by counting the number of cells (invasion, $n=3$ ) or tumour foci (metastasis, $n=5$ ) in five randomly selected high-power fields using the microscope (right panel). The data are presented as mean \pm SEM. 
A

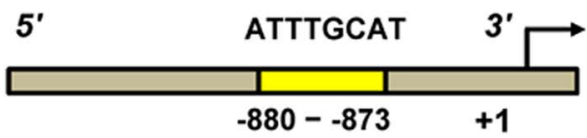

C

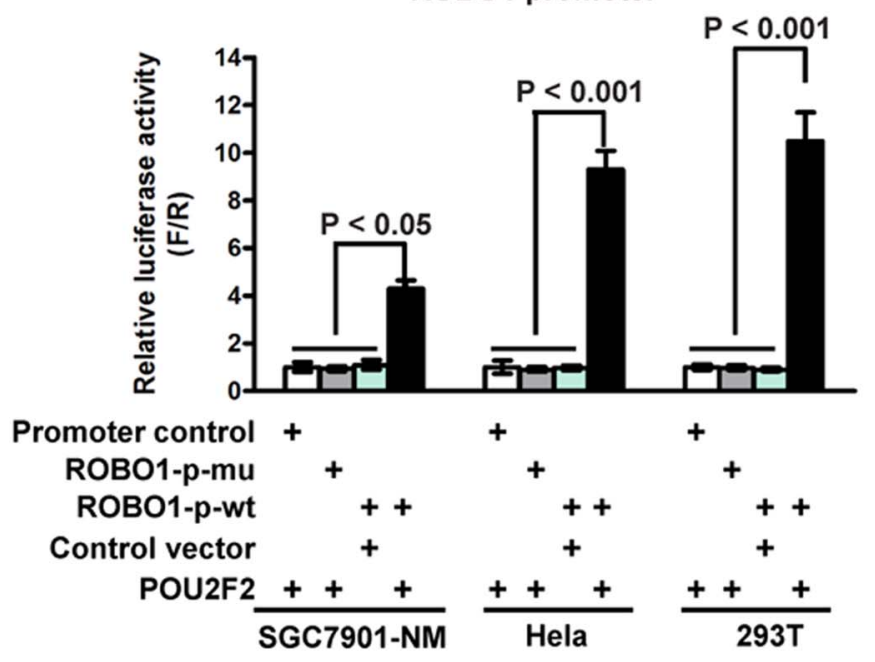

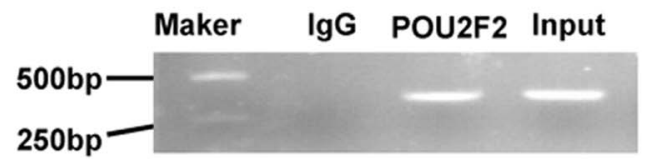

D

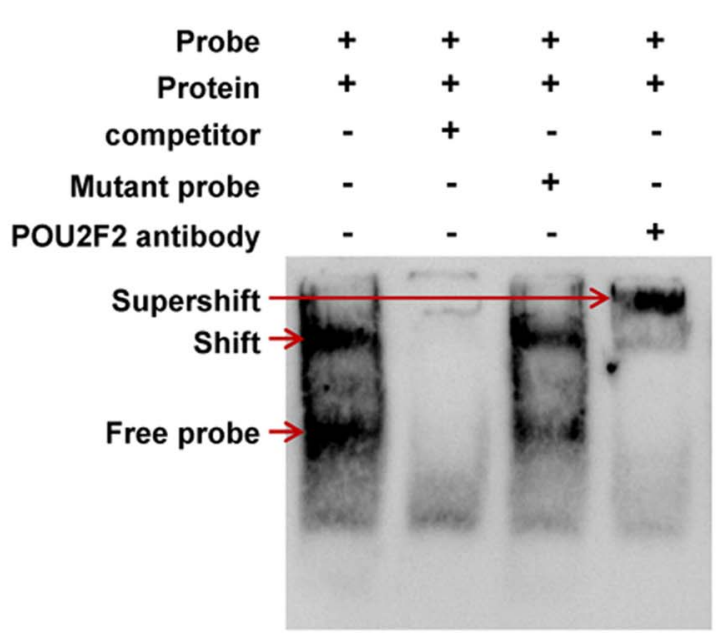

E

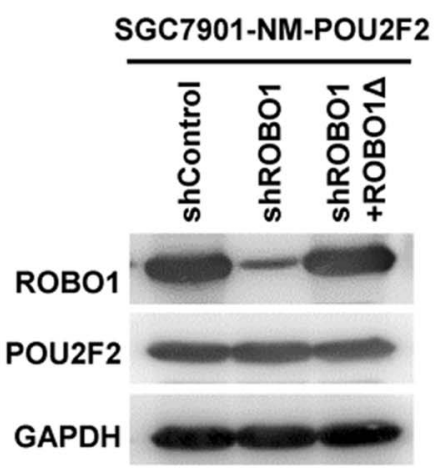

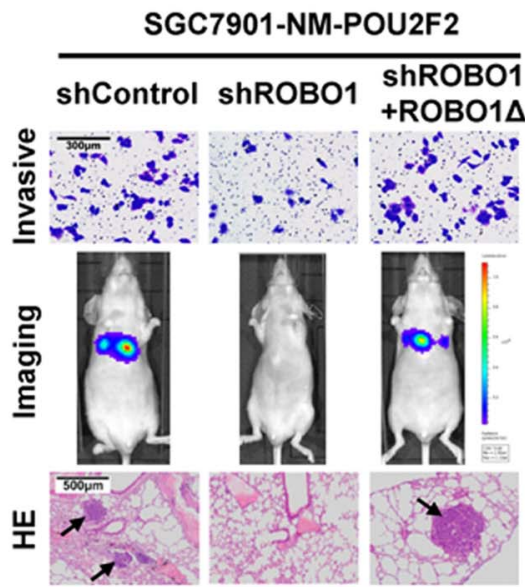
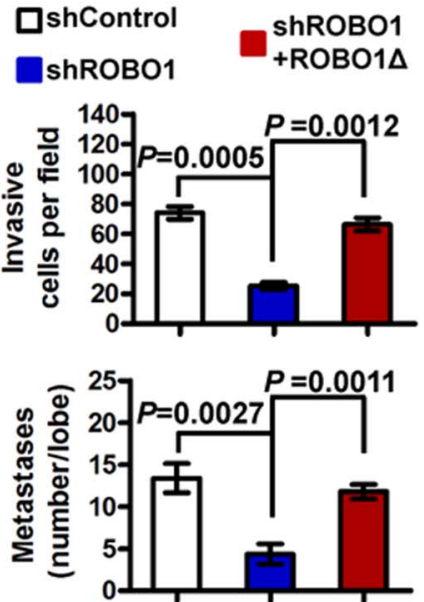

Figure 3 POU2F2 positively regulates ROBO1 transcription. (A) Schematic diagram of the ROBO1 promoter. The yellow region is the putative POU2F2-binding site in the ROBO1 promoter. (B) Amplification of the ROBO1 promoter sequence from chromatin immunoprecipitation (ChIP) DNA was performed. Input and IgG served as positive and negative controls, respectively. The electrophoresis results of PCR products confirmed that POU2F2 binds to the ROBO2 promoter. (C) Reporter gene analysis of the transcriptional activation ability of the ROBO1 promoter. The ROBO1 full-length promoter reporter gene was co-transfected with the POU2F2 gene expression vector into SGC7901-NM, HeLa and 293T cells, and the luciferase activities were measured. Selective mutagenesis at position -880 to $-873 \mathrm{bp}$ from the transcription start site lost response to POU2F2, indicating that this region is a POU2F2-responsive region $(n=3)$. (D) Electrophoretic mobility shift assay (EMSA) identified the POU2F2-binding site in the ROBO1 promoter. Nuclear protein was extracted from SGC7901-M cells and incubated with a biotin-labelled DNA probe in preparation for chemiluminescent EMSA. The unlabelled probe and its mutant probe were used as the cold competitor and negative control, respectively. A POU2F2-specific antibody was used to block the DNA complex shift with the aim of confirming the specificity of the POU2F2 binding motif. The arrow points to the POU2F2 DNA-binding complex. (E) Downregulated ROBO1 expression in SGC7901-NM-POU2F2 cells inhibited cell invasion and metastasis induced by POU2F2 overexpression. A shROBO1-resistant reconstitution, ROBO1 $\triangle$, was used to restore ROBO1 expression, which recovered the high metastatic ability of the cells.

regulated ROBO1 expression via this binding site and that this was the only binding site (figure 3C). A probe was synthesised based on this binding site, and EMSA was performed, which further confirmed that POU2F2 may bind to this site (figure 3D). The results suggested that POU2F2 positively regulates ROBO1 expression in GC cells.
ROBO1-specific siRNAs were transfected in SGC7901-M cells. According to the western blot analysis, siRNA4 had the highest inhibitory rate and was selected to construct the lentiviral expression vector, shROBO1 (see online supplementary figure S4). Downregulating the expression of $\mathrm{ROBO} 1$ in SGC7901-NM-POU2F2 cells obstructed the cell invasion and 
metastasis induced by POU2F2 overexpression. A mutant shROBO1-resistant lentiviral vector was then used to restore ROBO1 expression, which recovered the invasion and metastatic ability of the cells (figure $3 \mathrm{E}$ ). All of the data indicated that ROBO1 serves as a key target gene in GC metastasis induced by POU2F2 overexpression.

ROBO1 ligands are comprised of SLIT1, SLIT2 and SLIT3. SLIT1 expression is confined to the nervous system, and SLIT3 is downregulated in GC. ${ }^{8}$ Therefore, we hypothesised that the interaction between SLIT2 and ROBO1 stimulates GC metastasis. In addition, RNAi-mediated silencing of SLIT2 decreased tumour invasion and metastasis driven by ROBO1 overexpression (see online supplementary figure S5). These findings suggested that ROBO1-mediated POU2F2 overexpression promotes GC metastasis in a SLIT2-dependent manner.

Epithelial-mesenchymal transition (EMT) endows epithelial cells with interstitial features, and it is closely correlates with tumour cell invasion and metastasis. ${ }^{31} 32$ Therefore, we investigated whether POU2F2 could link EMT with tumour metastasis. We first measured the expression status of several renowned EMT markers ( $\beta$-catenin, E-cadherin, vimentin, MMP2 and MMP9) in the SGC7901-M-siControl, SGC7901-M-siPOU2F2, SGC7901-NM-Control and SGC7901-NM-POU2F2 cells via western blot analysis and immunofluorescence staining. The POU2F2-overexpressing cells exhibited an elevated expression of Vimentin, MMP2 and MMP9, but these cells had a reduced level of nuclear $\beta$-catenin and E-cadherin. Moreover, we also observed EMT phenotypes in POU2F2-overexpressing cells (see online supplementary figure S6). These findings implied a possible pro-metastatic role of EMT in POU2F2-overexpressing cells.

\section{POU2F2 is transcriptionally activated by NF- $\mathrm{kB}$ in GC cells}

Previous studies have found that POU2F2 expression in Hodgkin/Reed-Sternberg cells, ${ }^{13}$ precursor B lymphocytes ${ }^{14}$ and pancreatic cancer cells ${ }^{6}$ highly correlates with the activity of $\mathrm{NF}-\kappa \mathrm{B}$, suggesting that the POU2F2 gene may be an NF- $\mathrm{B}$ target gene. Based on these previous findings, we tested whether $\mathrm{NF}-\mathrm{\kappa B}$ directly activates POU2F2 transcription in GC, thus leading to POU2F2 overexpression. To evaluate this hypothesis, we transfected SGC7901-NM cells with an empty vector or pBabe-Puro-IKBalpha-mut (super repressor) encoding a mutant $\mathrm{I} \kappa \mathrm{B} \alpha$, which is resistant to stimulus-induced degradation and NF- $\mathrm{BB}$ activation. The SGC7901-NM-vector cells were then treated with six different concentrations $(0-15 \mu \mathrm{g} / \mathrm{mL})$ of lipo-

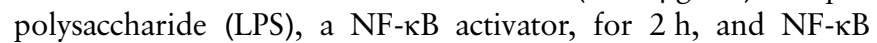
activity was detected using a NF- $\mathrm{B}$ activity reporter gene. Within the LPS concentration range of $0-10 \mu \mathrm{g} / \mathrm{mL}$, NF- $\kappa B$ activity was increased in a dose-dependent manner. The cells were treated with $10 \mu \mathrm{g} / \mathrm{mL}$ LPS, and NF- $\mathrm{BB}$ activity was examined at $0,1,2,4$ and $6 \mathrm{~h}$. The results showed that the NF- $\kappa \mathrm{B}$ activity reached a peak at $4 \mathrm{~h}$, and the expression of POU2F2 and its target gene, ROBO1, was upregulated with the enhanced activity of NF- $\mathrm{KB}$ in SGC7901-NM-vector cells. However, the activity of NF- $\mathrm{NB}$, along with POU2F2 and ROBO1 expressions, revealed no change in SGC7901-NM-IкB $\alpha$-mut cells (figure $4 \mathrm{~A}$ ). NF- $\mathrm{\kappa B}$ was predicted to have four potential binding sites in the POU2F2 promoter region based on the TRANSFAC data and Promoter 2.0 (http://www.cbs.dtu.dk/services/ Promoter/). Reporter genes for truncated bodies containing different binding sites were constructed with a reduced-order method, and the data indicated that POU2F2 was transcriptionally activated by $\mathrm{NF}-\kappa \mathrm{B}$ and the regulation region of $\mathrm{NF}-\kappa \mathrm{B}$ was located in the POU2F2 promoter region at -600 to $-300 \mathrm{bp}$ (figure 4B). The wild-type POU2F2 full-length promoter reporter gene and the NF- $\mathrm{kB}$ binding site mutant promoter reporter gene were transfected into SGC7901-NM, MKN28-NM and 293T cells, and the activity of the reporter genes was monitored after LPS stimulation. The results showed that the regulation of POU2F2 by $\mathrm{NF}-\mathrm{KB}$ disappeared with the locus mutation of 531 to $-522 \mathrm{bp}$ (figure $4 \mathrm{C}$ ). The primers and probes were designed based on this binding site for ChIP and EMSA experiments, and we observed that the both subunits of $\mathrm{NF}-\kappa \mathrm{B}, \mathrm{p} 65$ and p50, directly bound to the site (figure 4D, E, see online supplementary figure S7). In summary, POU2F2 overexpression in GC cells may be associated with the activation of $\mathrm{NF}-\kappa \mathrm{B}$ signalling pathways.

\section{POU2F2 links NF-кB to the SLIT2/ROBO1 signalling pathway contributing to $\mathrm{GC}$ metastasis}

In the present report, we demonstrated that the excessive activation of NF- $\mathrm{KB}$ induced POU2F2 overexpression. Meanwhile, increased levels of POU2F2 upregulated the expression of the membrane receptor, ROBO1, and activated the SLIT2/ ROBO1 signalling pathway, thus stimulating GC cell metastasis. These data suggested that POU2F2 might be the key to connect $\mathrm{NF}-\kappa \mathrm{B}$ and the SLIT2/ROBO1 signalling pathways in GC metastasis. To test whether the POU2F2-mediated interaction of the $\mathrm{NF}-\kappa \mathrm{B}$ and SLIT2/ROBO1 signalling pathways is the main mechanism of GC metastasis with POU2F2 high expression, SGC7901-NM cells were treated with $10 \mu \mathrm{g} / \mathrm{mL}$ LPS for $4 \mathrm{~h}$. The results showed that NF- $\mathrm{BB}$ activity was significantly augmented. The POU2F2 and ROBO1 levels were also robustly increased. Moreover, the cell invasion and metastasis capacity was enhanced. All of these effects induced by LPS were blocked by $\mathrm{pBabe}$-Puro-IKBalpha-mut, thereby indicating that the LPS-imposed effects on GC cells, including POU2F2 upregulation, ROBO1 upregulation and increased cell metastasis, are NF-кB-dependent (figure 5A, see online supplementary figure S8). When POU2F2 expression was downregulated by shRNA, the elevated expression of ROBO1 and cell metastasis ability due to the activation of NF- $\mathrm{BB}$ was decreased. Exogenous overexpression of ROBO1 antagonised the effects of shPOU2F2 on GC cell invasion and metastasis (figure 5B). The results suggested that the interaction of the NF- $\mathrm{BB}$ signalling pathway with SLIT2/ROBO1 signalling may serve as a main mechanism of GC metastasis and that POU2F2 may be the link between these two pathways. We assessed the correlation of the expression of P65, POU2F2 and ROBO1 in 266 cases of GC tissues by immunohistochemistry. As shown in figure $5 \mathrm{C}$, the overlapping expression of all three molecules was observed in 50.75\% (135/266) of GC tissues and $70.50 \%(141 / 200)$ of GC tissues with lymph node or distant metastasis, thus suggesting a close association between their expression changes in GC, especially in metastatic GC.

\section{miR-218 suppresses GC metastasis by inhibiting multiple} genes in the NF-кB/POU2F2/ROBO1 signalling pathway

In addition to the transcriptional regulation by NF- $\mathrm{B}$, posttranscriptional regulation involving miRNAs also contributes to POU2F2 expression. TargetScanHuman 6.2 (http://www. targetscan.org/) analysis indicated that the POU2F2 3'-UTR region contained miR-218-binding sites, suggesting that POU2F2 might be the target gene of miR-218. Previous studies have reported that miR-218 may directly regulate $\mathrm{ROBO} 1^{8}$ and the IKK- $\beta$ kinase in the NF- $\kappa \mathrm{B}$ signalling pathway. ${ }^{27}$ To confirm the simultaneous regulation of POU2F2, IKK- $\beta$ and ROBO1 by miR-218, we constructed the $3^{\prime}$-UTR reporter genes for IKK- $\beta$, POU2F2 and ROBO1-containing miR-218 binding sites as well 

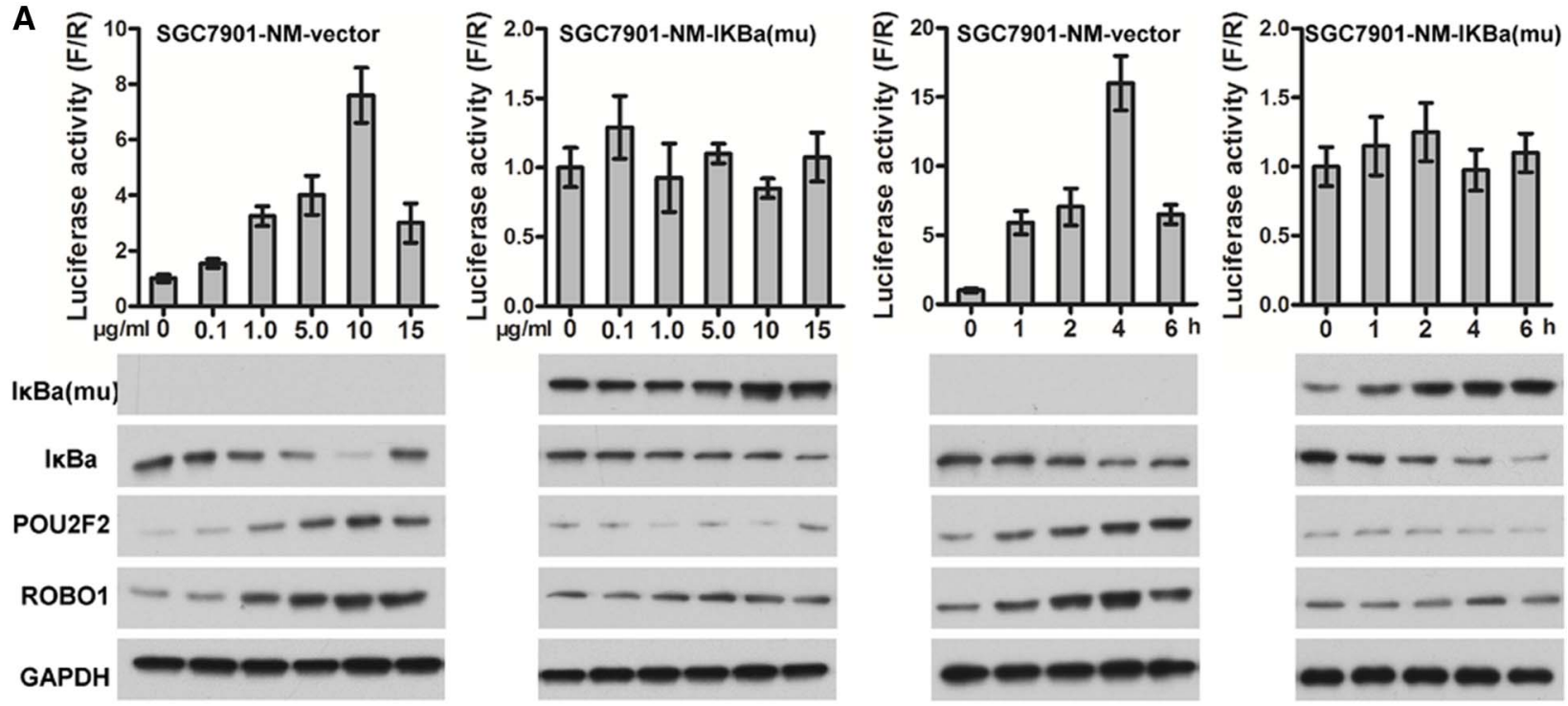

B

Putative NF-kB binding sites

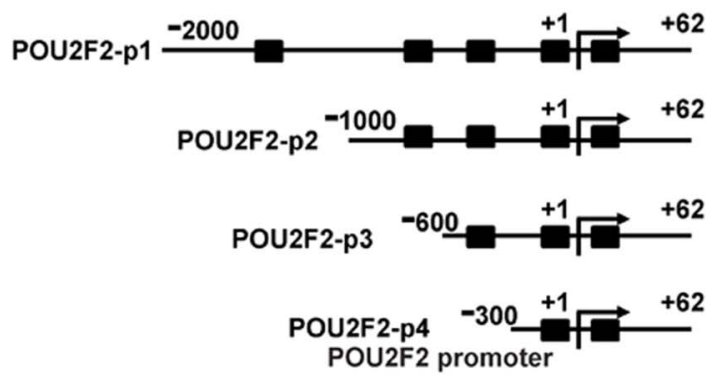

C

POU2F2 promoter

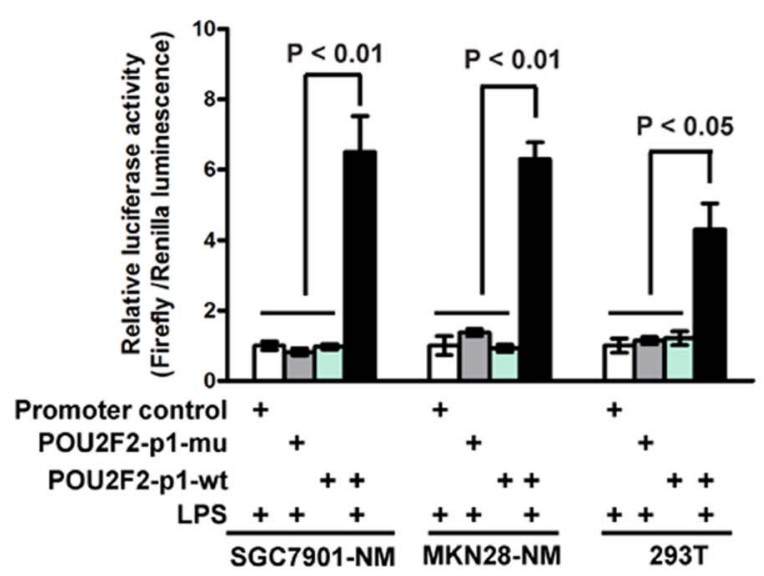

Relative luciferase activity (F/R)
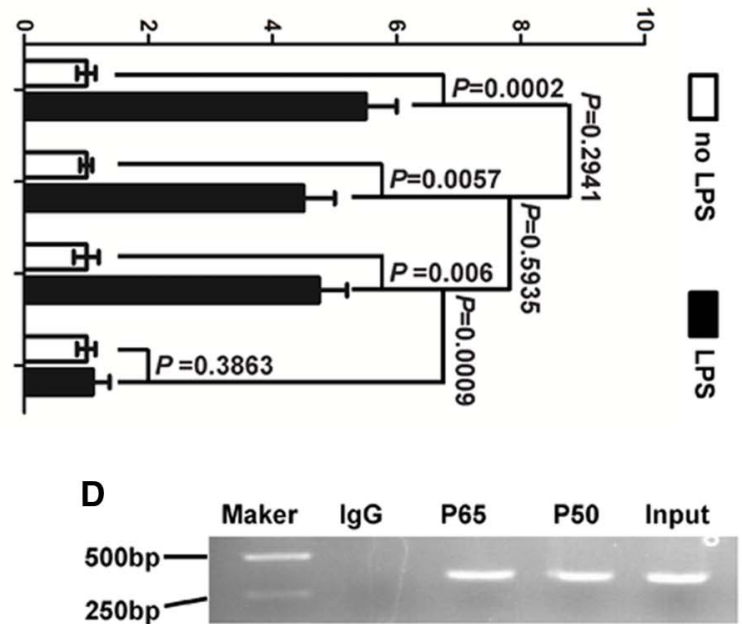

E

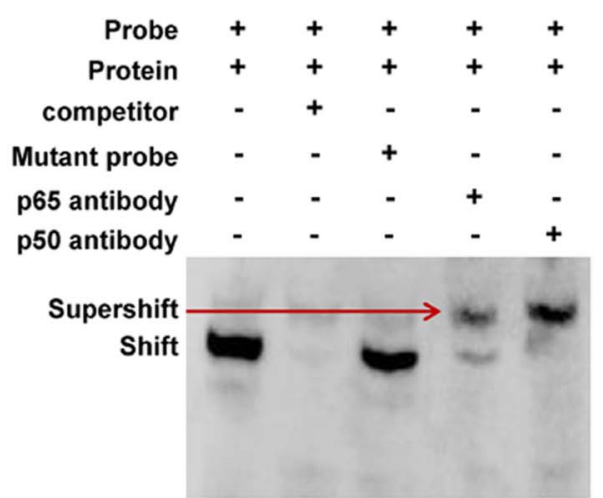

Figure 4 POU2F2 is induced by activated nuclear factor (NF)- $\kappa B$ in gastric cancer (GC). (A) The POU2F2 expression was positively related with NF- $\kappa B$ activity. SGC7901-NM was transduced with the pBabe-Puro-IKBalpha-mut vector encoding a NF- $\kappa B$-specific inhibitor or an empty vector. Cells were stimulated for $2 \mathrm{~h}$ with different doses of lipopolysaccharide (LPS) or for different time with $10 \mu \mathrm{g} / \mathrm{mL} \mathrm{LPS}$. NF- $\mathrm{KB}$ activity (upper panel) was detected by a reporter gene. IкBa-mu, IкBa, POU2F2 and ROBO1 expressions (lower panel) were examined by western blot assays. The expressions of POU2F2 and ROBO1 were positively related with NF- $\kappa B$ activity in SGC7901-NM-vector. NF- $\kappa B$ activity, as well as the expressions of POU2F2 and ROB01, was almost no change in SGC7901-NM-IKBalpha-mut cells $(n=3)$. (B) The truncation reporter genes containing different binding sites were used to analyse the NF- $\kappa B$ transcription activity sites in the POU2F2 promoter region. The regulatory region of NF- $\kappa B$ was located at the -600 to $-300 \mathrm{bp}$ region in the POU2F2 promoter $(n=3)$. (C) The POUF2F wild-type and the POU2F2 -531 to -522 bp mutation reporter genes were used to analyse the binding sites of NF-KB in the POU2F2 promoter $(n=3)$. (D) In vivo chromatin immunoprecipitation (ChIP) experiments validated the binding capacity of NF- $K B$ to the POU2F2 promoter. (E) In vivo electrophoretic mobility shift assay (EMSA) experiments verified the binding sequence of NF- $\kappa B$ in the POU2F2 promoter in the 531 to -522 bp region $(n=3)$. 
A
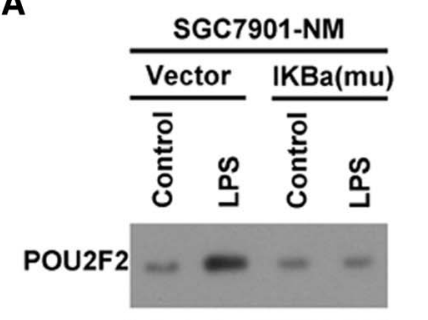

ROBO1

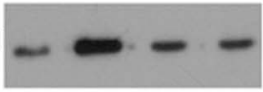

GAPDH

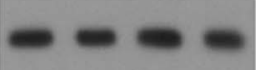

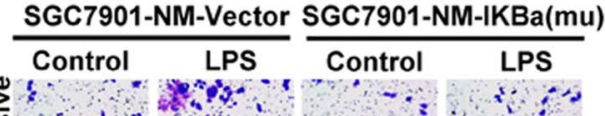

要

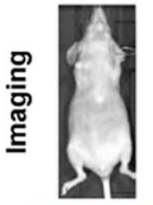

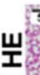

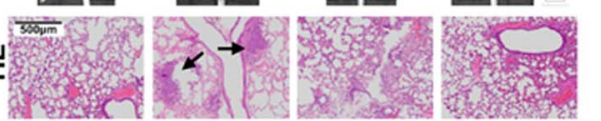

$\square$ Vector+Control $\square$ Vector+LPS

口IKBa(mu)+Control $\square$ IKBa(mu)+LPS
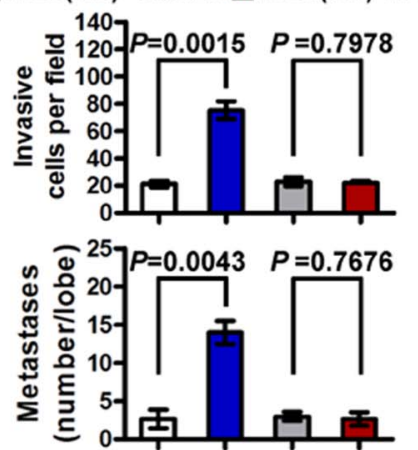

B

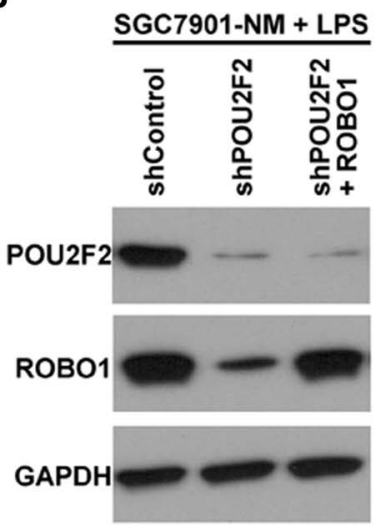

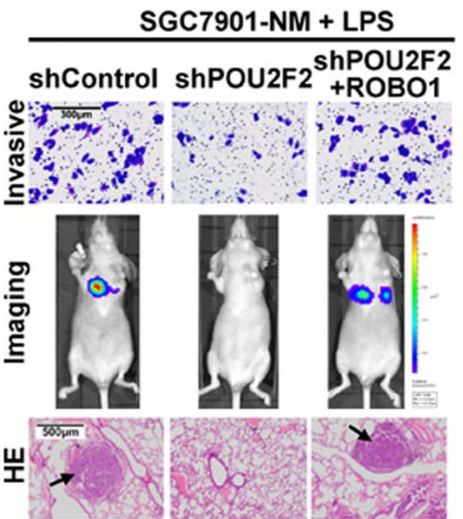
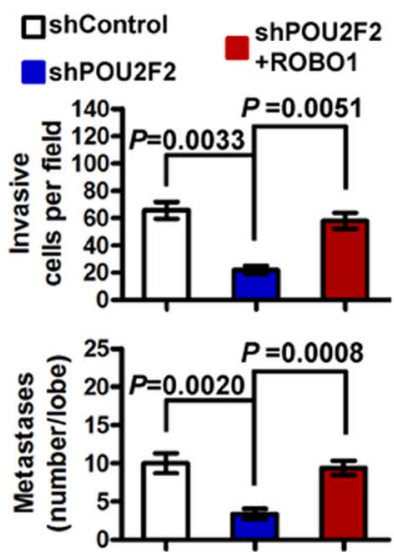

C

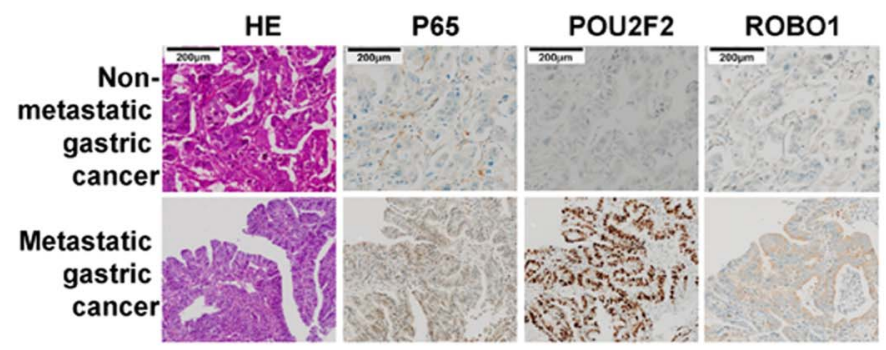

Gastric cancer

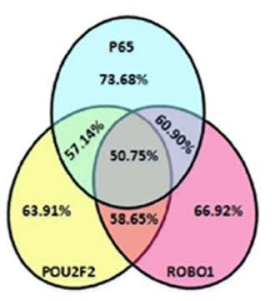

Metastatic gastric cancer

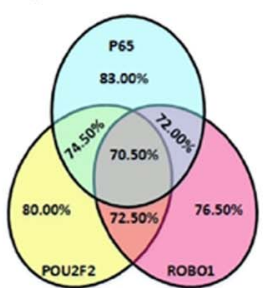

Figure 5 POU2F2 links nuclear factor (NF)- $\mathrm{kB}$ and the SLIT2/ROB01 signalling pathway to promote gastric cancer (GC) metastasis. (A) NF- $\mathrm{kB}$ inhibition reversed lipopolysaccharide (LPS)-induced tumour invasion and metastasis. In LPS-induced SGC7901-NM-vector cells, as the NF-KB activity enhanced, the expressions of POU2F2 and ROBO1 as well as the cell invasion and metastasis capacity correspondingly increased. However, in SGC7901-NM- IкBa-mu cells in which NF-кB was inhibited by IкBa-mu, no changes were observed. (B) Downregulated POU2F2 expression blocked increase of ROBO1 expression induced by NF-KB activation, thus reducing cell invasion and metastasis. The exogenous overexpression of ROBO1 antagonised the effects of POU2F2 shRNA on the invasion and metastasis of GC cells. (C) The expressions of P65, POU2F2 and ROBO1 in GC were detected by immunohistochemical methods (left). A Venn diagram shows the correlation among the three genes in all GCs $(n=266)$ and metastatic $\mathrm{GCs}(\mathrm{n}=200$; right).

as the reporter genes for the binding site mutations, and we co-transfected the miR-218 vectors into SGC7901-M and MKN28-M cells. The activity of the wild-type reporter genes was dramatically impaired, but there was no noticeable change in the activity of mutated reporter genes (figure 6A). The upregulation of miR-218 expression in SGC7901-M and MKN28-M cells using the miR-218 lentiviral vector resulted in decreased protein expression of IKK- $\beta$, POU2F2 and ROBO1 as well as reduced cell invasion and metastasis. In contrast, using miR-218 inhibitors to suppress miR-218 expression in SGC7901-NM and MKN28-NM cells, the opposite results were observed (figure 6B, C, see online supplementary figures $\mathrm{S} 9$ and $\mathrm{S} 10$ ). In addition, using shRNA to repress the expression of these three genes resulted in a metastatic phenotype similar to that resulting from miR-218 overexpression (figure 6D, see online supplementary figure S10). In contrast, using the expression vector without miR-218-binding sites to enhance the levels of IKK- $\beta$, POU2F2 or ROBO1 in MKN28-M-miR-218 cells resulted in the partial rescue of the miR-218-mediated inhibition of GC metastasis (figure 6E). Our findings indicated that miR-218 suppresses GC metastasis by inhibiting multiple genes in the NF-KB/POU2F2/ ROBO1 signalling pathway. 


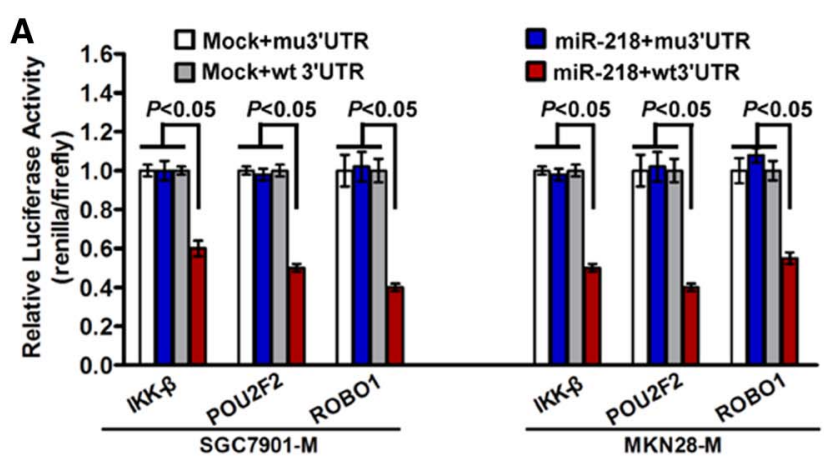

B

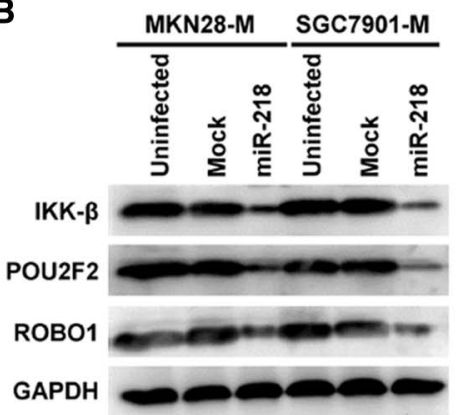

D
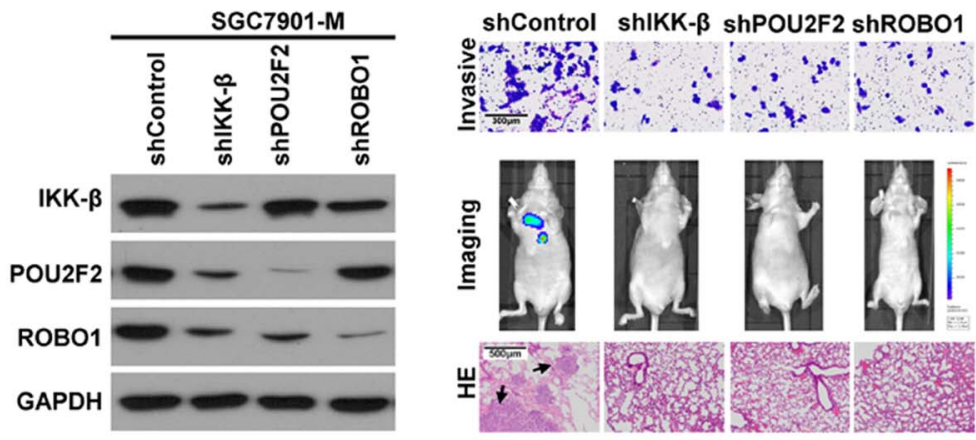

E
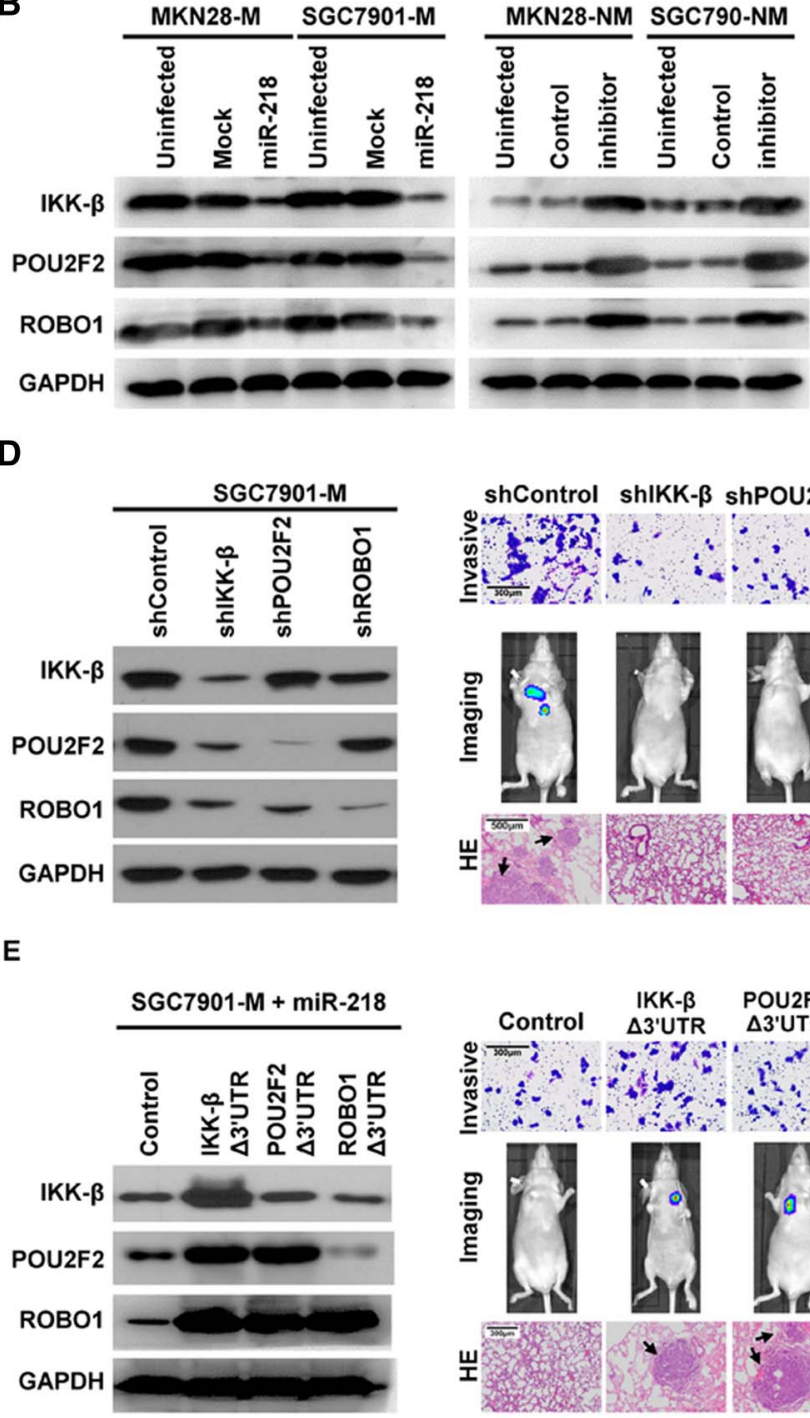

C

SGC7901-M SGC7901-NM
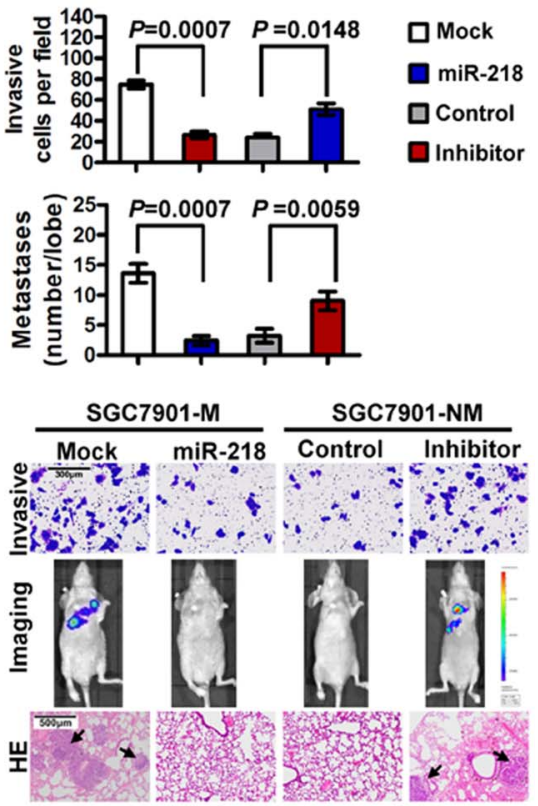

Figure 6 miR-218 suppresses gastric cancer (GC) metastasis by simultaneous inhibiting nuclear factor (NF)- $\kappa B$ activity, POU2F2 expression and ROB01 expression. (A) miR-218 luciferase reporter assay. The wild-type and mutant miR-218 targets (IKK- $\beta$, POU2F2 and ROBO1) were fused with a luciferase reporter and transfected into mock-infected or miR-218-infected SGC7901-M and MKN28-M cells. miR-218 significantly suppressed the luciferase activity of the wild-type luciferase reporters $(n=3)$. (B) Upregulated expression of miR-218 resulted in downregulated protein expression of IKK- $\beta$, POU2F2 and ROB01. Downregulated expression of miR-218 resulted in the opposite changes. (C) miR-218 negatively regulated the invasion and metastasis of GC cells. (D) Downregulating the expression of IKK- $\beta$, POU2F2 or ROBO1 led to phenotypic changes similar to miR-218 overexpression. (E) The overexpression of IKK- $\beta$, POU2F2 or ROBO1 antagonised the inhibition of the metastatic phenotypes of GC cells induced by miR-218.

\section{DISCUSSION}

This study determined the specific expression of POU2F2 in metastatic GC and its key role in promoting GC metastasis. Both the transcriptional activation of $\mathrm{NF}-\mathrm{\kappa B}$ and post-transcriptional repression degradation of miR-218 gave rise to POU2F2 expression. NF- $\kappa \mathrm{B}$ directly regulated POU2F2, and POU2F2 directly targeted ROBO1. Therefore, POU2F2 links NF- $\mathrm{BB}$ to the SLIT2/ ROBO1 signalling pathway. The interaction of $\mathrm{NF}-\kappa \mathrm{B}$ with the 
POU2F2/SLIT2/ROBO1 pathway advocates GC metastasis. In addition, miR-218 inhibited GC metastasis by simultaneously repressing multiple target molecules in this interacting network.

POU2F2 has been previously thought to be only expressed in $\mathrm{B}$ cells and B-cell-derived tumour cells. ${ }^{2-4}$ Hippo et $a l^{7}$ discovered a high and specific expression of POU2F2 in metastatic GC tissue, but the molecular mechanisms underlying its effect in GC were far from clear. This study not only reaffirmed the increased expression of POU2F2 in metastatic GC but, more importantly, it also revealed the role of POU2F2 in conferring GC cell metastasis through a direct regulation of ROBO1 and the activation of SLIT2/ROBO1 signalling. SLIT2/ROBO1 signalling, a critical signalling pathway in the development of the nervous system, is capable of modulating oriented axon growth and neuronal migration exclusion. ${ }^{33}$ Recently, a growing number of studies have reported an increase of SLIT2/ROBO1 expression in tumour tissues, and they have even described the role SLIT2/ROBO1 in promoting tumorigenesis and metastasis. ${ }^{9} 103536$ We have identified ROBO1 overexpression in GC cells, especially in GC cells with high metastatic potential. ${ }^{8}$ However, our understanding of abnormal ROBO1 expression in GC remains unclear. Here, for the first time, we discovered and confirmed that through direct binding to the ROBO1 promoter region at sites -880 to -873 , POU2F2 transcriptionally activates ROBO1, thus stimulating the SLIT2/ROBO1 signalling pathway and ultimately promoting GC metastasis. We found that POU2F2 overexpression promoted GC metastasis via SLIT2/ROBO1 signalling. Meanwhile, GC cells overexpressing POU2F2 exhibited a phenotype of EMT, but whether it is associated with the activation of SLIT2/ROBO1 pathway still needed to be further verified.

The SLIT2/ROBO1 pathway does not work via the same downstream signalling in different cell types. Much is known about the ROBO1 downstream signalling mechanisms that mediate cell migration. For instance, Wang et al ${ }^{10}$ demonstrated that PI-3K is involved in endothelial cell responses to SLIT2/ ROBO1 interaction. In neuronal cells, Bashaw et $a l^{37}$ demonstrated that Abelson and Enabled are downstream targets of the SLIT2/ROBO1 pathway, and Guerrier et $a l^{38}$ suggested that
srGAP2 plays direct roles in ROBO1 signal transduction. Considering all these findings, the extent to which SLIT2/ ROBO1 specifically affects GC metastasis still remains unclear. However, our results suggested that SLIT2/ROBO1 signalling functions as a crucial modulator to stimulate GC metastasis. However, some studies have also suggested that SLIT2/ROBO1 signalling acts as a tumour suppressor. ${ }^{39-41}$ Possible explanations for these differences include different tumour origins and various metastatic mechanisms. For example, p21, a tumour suppressor in GC and colorectal cancer, serves as a tumour promoter in prostate and ovarian cancers. ${ }^{42}$ Therefore, SLIT2/ ROBO1 signalling may not play the same roles in different types of cancer or even in different stages of the same cancer type. Previous studies by Poo and colleagues have shown that the same guidance cue can either attract or repel the same axons depending on intracellular cyclic adenosine monophosphate (cAMP) and cyclic guanosine monophosphate (cGMP) levels. ${ }^{43}$ Thus, the determination of the exact role that cAMP and cGMP play in cancer metastasis may be helpful to explain the existing contradiction of SLIT2/ROBO1 functions.

Our findings, for the first time, indicated that the abnormal expression of POU2F2 in GC cells with high metastatic potential might be due, at least in part, to the activation of NF- $\kappa$ B. Our study was the first to confirm in GC cells that by binding to POU2F2 promoter sites -531 to $-522 \mathrm{bp} \mathrm{NF- \kappa B}$ transcriptionally regulates POU2F2 expression, resulting in an interaction network with POU2F2 in the centre. This network involves upstream regulation by $\mathrm{NF}-\kappa \mathrm{B}$, POU2F2 and the downstream molecule, ROBO1. It is important to further investigate the contributions of this interacting network in neurons, endothelial cells and other tumour cells.

In addition to transcriptional regulation by $\mathrm{NF}-\kappa \mathrm{B}$, posttranscriptional regulation by $\mathrm{miR}-218$ is also a major cause for aberrant POU2F2 expression. Our study is the first to not only uncover the direct regulation of POU2F2 by miR-218 but to also demonstrate that miR-218 directly mediates its upstream regulator, NF- $\kappa \mathrm{B}$-kinase $\beta$ (IKK- $\beta$ ), and the POU2F2 downstream target gene, ROBO1. Our previous studies have shown that miR-218 levels decrease with decreasing expression levels
Figure 7 A new SLIT/miR-218/

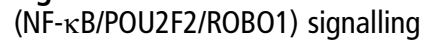
pathway promotes gastric cancer (GC) metastasis. During GC development, change in SLIT3 gene expression decreases the expression of miR-218, which removes the inhibitory effect on the translation of IKK- $\beta$, POU2F2 and ROB01. The expressions of IKK- $\beta$, POU2F2, and ROBO1 are upregulated. In particular, the upregulated IKK- $\beta$ activates nuclear factor (NF)- $\kappa B$, which further promotes the upregulation of POU2F2 and ROBO1. ROBO1 interacts with the SLIT2 ligand, which activates the SLIT2/ROBO1 signalling pathway, thus promoting $\mathrm{GC}$ metastasis.

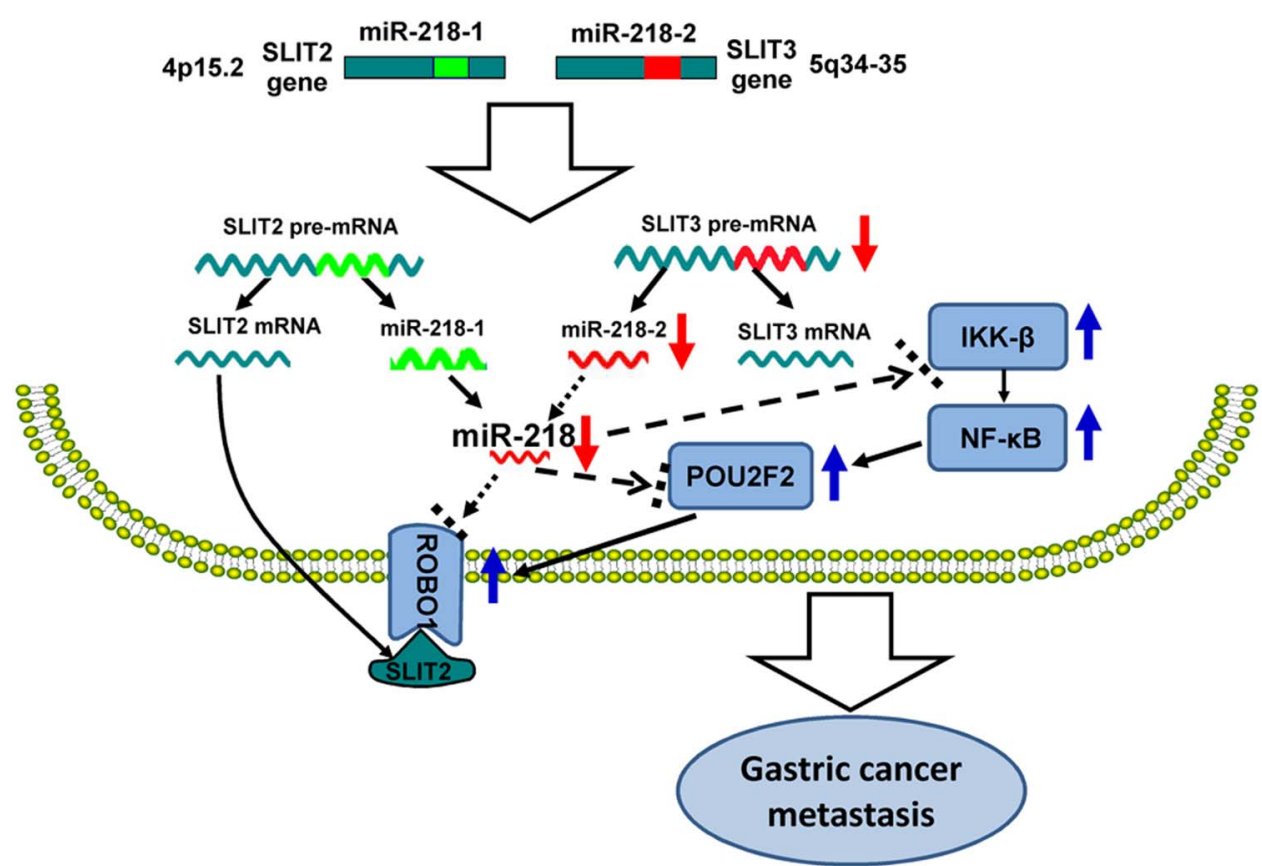


of SLIT3, one of its host genes, in metastatic GC. Decreased miR-218 eliminates ROBO1 repression, resulting in ROBO1 upregulation. Increased ROBO1 interacts with SLIT2, one of its ligands, to trigger tumour metastasis owing to the activation of the SLIT/ROBO1 pathway. Ectopic expression of miR-218 suppresses GC metastasis by mediating the SLIT2/ROBO1 signalling pathway. Based on these findings, we have suggested that a SLIT/miR-218/ROBO1 signalling pathway exists in GC, ${ }^{8}$ which has been verified not only in other tumours types but also in human tissues and organs by researchers worldwide. ${ }^{21}{ }^{23-26}$ In the present study, this regulation loop was further expanded (figure 7). By way of correlative studies between expression and function, we validated that in addition to ROBO1 miR-218 also simultaneously inhibits IKK- $\beta$ and POU2F2 genes. Under the simultaneous regulation of these three target genes, GC metastasis is synergistically inhibited. These results provided further insight into the underlying mechanisms of miR-218. More importantly, due to its regulative effects on various GC metastasis-associated genes and signalling pathways, miR-218 has higher priority than a single protein-coding gene in GC gene therapy.

Collectively, the findings of this study revealed the tumourpromoting role that POU2F2 plays in GC metastasis and the mechanisms of POU2F2 facilitating GC metastasis through regulating ROBO1. Moreover, we identified the molecular mechanisms of NF- $\mathrm{KB}$ activation-induced expression of POU2F2, and we discovered that the POUF2F-mediated interaction between $\mathrm{NF}-\mathrm{KB}$ and the SLIT2/ROBO1 signalling pathway contributes to GC metastasis. Ultimately, miR-218 can simultaneously orchestrate multiple molecules in the two interacting signalling pathways and is a potential target for therapy.

Acknowledgements We wish to thank Prof. Zhang Bo (Department of Pathology, Fourth Military Medical University) for valuable comments on the manuscript, and we would like to thank Dr Xia CaiHao for technical assistance.

Contributors SMW, JT, WLW and SJH contributed equally. D-MF and JT designed the study. JT, S-MW, WLW, SJH, JPY, XFY, ZHT and XYZ performed the experiments. JT and S-MW analysed the data and wrote the manuscript. YZN and ZSL evaluated the histological features. MBL, YZN and KCW contributed reagents and materials.

Funding This research was supported by the National Science Foundation of China (no. 81272649 no. 81227901, no. 81225003 and no. 81430072) and the National Key and Basic Research Development Program of China (no. 2010CB529302 and Major Projects of Ministry of Science and Technology (2012ZX09303011-001)). The funders had no role in study design, data collection, data analysis, decision to publish or preparation of the manuscript.

Competing interests None declared.

Patient consent Obtained.

Ethics approval All experimental procedures were approved by the Institutional Review Board of Xijing Hospital. Animal experiments were carried out with the approval of the Institutional Committee for Animal Research and in accordance with national guidelines for the care and use of laboratory animals.

Provenance and peer review Not commissioned; externally peer reviewed.

Open Access This is an Open Access article distributed in accordance with the Creative Commons Attribution Non Commercial (CC BY-NC 4.0) license, which permits others to distribute, remix, adapt, build upon this work non-commercially, and license their derivative works on different terms, provided the original work is properly cited and the use is non-commercial. See: http://creativecommons.org/ licenses/by-nc/4.0/

\section{REFERENCES}

1 Friedl EM, Matthias P. Transcriptional activation and repression, two properties of the lymphoid-specific transcription factor Oct-2a. Eur J Biochem 1995;234:308-16.

2 Robinson AR, Kwek SS, Kenney SC. The B-cell specific transcription factor, Oct-2, promotes Epstein-Barr virus latency by inhibiting the viral immediate-early protein, BZLF1. PLoS Pathog 2012;8:e1002516.

3 Saito M, Tanaka S, Mori A, et al. Primary gastric Hodgkin's lymphoma expressing a B-Cell profile including Oct-2 and Bob-1 proteins. Int J Hematol 2007;85:421-5.
4 Garcia-Cosio M, Santon A, Martin P, et al. Analysis of transcription factor OCT.1, OCT.2 and BOB.1 expression using tissue arrays in classical Hodgkin's lymphoma. Mod Pathol 2004;17:1531-8.

5 Malone CS, Patrone L, Buchanan KL, et al. An upstream Oct-1- and Oct-2-binding silencer governs B29 (Ig beta) gene expression. J Immunol 2000;164:2550-6.

6 Marin-Muller C, Li D, Bharadwaj U, et al. A tumorigenic factor interactome connected through tumor suppressor microRNA-198 in human pancreatic cancer. Clin Cancer Res 2013;19:5901-13.

7 Hippo Y, Taniguchi H, Tsutsumi S, et al. Global gene expression analysis of gastric cancer by oligonucleotide microarrays. Cancer Res 2002;62:233-40.

8 Tie J, Pan Y, Zhao L, et al. MiR-218 inhibits invasion and metastasis of gastric cancer by targeting the Robo1 receptor. PLoS Genet 2010;6:e1000879.

9 Mertsch S, Schmitz N, Jeibmann A, et al Slit2 involvement in glioma cell migration is mediated by Robo1 receptor. J Neurooncol 2008;87:1-7.

10 Wang B, Xiao Y, Ding B-B, et al. Induction of tumor angiogenesis by Slit-Robo signaling and inhibition of cancer growth by blocking Robo activity. Cancer Cell 2003;4:19-29.

11 Brose K, Tessier-Lavigne M. Slit proteins: key regulators of axon guidance, axonal branching, and cell migration. Curr Opin Neurobiol 2000;10:95-102.

12 Wu JY, Feng L, Park HT, et al. The neuronal repellent Slit inhibits leukocyte chemotaxis induced by chemotactic factors. Nature 2001;410:948-52.

13 Bargou RC, Leng C, Krappmann D, et al. High-level nuclear NF-kappa B and Oct-2 is a common feature of cultured Hodgkin/Reed-Sternberg cells. Blood 1996:87:4340-7.

14 Bendall HH, Scherer DC, Edson CR, et al. Transcription factor NF-kappaB regulates inducible Oct-2 gene expression in precursor B lymphocytes. J Biol Chem 1997;272:28826-8.

15 Zhang $\mathrm{H}$, Li Y, Lai M. The microRNA network and tumor metastasis. Oncogene 2010;29:937-48

16 Wu W. MicroRNA: potential targets for the development of novel drugs? Drugs $R D$ 2010;10:1-8

17 Venkataraman S, Birks DK, Balakrishnan I, et al. MicroRNA 218 acts as a tumor suppressor by targeting multiple cancer phenotype-associated genes in medulloblastoma. J Biol Chem 2013;288:1918-28.

$18 \mathrm{Li} \mathrm{CH}$, To KF, Tong JH, et al. Enhancer of zeste homolog 2 silences microRNA-218 in human pancreatic ductal adenocarcinoma cells by inducing formation of heterochromatin. Gastroenterology 2013;144:1086-97 e9.

19 White NM, Fatoohi E, Metias M, et al. Metastamirs: a stepping stone towards improved cancer management. Nat Rev Clin Oncol 2011;8:75-84.

20 Uesugi A, Kozaki K, Tsuruta T, et al. The tumor suppressive microRNA miR-218 targets the mTOR component Rictor and inhibits AKT phosphorylation in oral cancer. Cancer Res 2011;71:5765-78.

21 Alajez NM, Lenarduzzi M, Ito E, et al. MiR-218 suppresses nasopharyngeal cancer progression through downregulation of survivin and the SLIT2-ROBO1 pathway. Cancer Res 2011;71:2381-91.

22 Wu DW, Cheng YW, Wang J, et al. Paxillin predicts survival and relapse in non-small cell lung cancer by microRNA-218 targeting. Cancer Res 2010;70:10392-401.

23 He $H$, Di Y, Liang $M$, et al. The microRNA-218 and ROBO-1 signaling axis correlates with the lymphatic metastasis of pancreatic cancer. Oncol Rep 2013;30:651-8

24 Guan H, Wei G, Wu J, et al. Down-regulation of miR-218-2 and its host gene SLIT3 cooperate to promote invasion and progression of thyroid cancer. J Clin Endocrinol Metab 2013;98:E1334-44.

25 Fish JE, Wythe JD, Xiao T, et al. A Slit/miR-218/Robo regulatory loop is required during heart tube formation in zebrafish. Development 2011;138:1409-19.

26 Small EM, Sutherland LB, Rajagopalan KN, et al. MicroRNA-218 regulates vascular patterning by modulation of Slit-Robo signaling. Circ Res 2010;107:1336-44.

27 Song L, Huang Q, Chen K, et al. miR-218 inhibits the invasive ability of glioma cells by direct downregulation of IKK-beta. Biochem Biophys Res Commun 2010;402:135-40.

28 Gao C, Zhang Z, Liu W, et al. Reduced microRNA-218 expression is associated with high nuclear factor kappa B activation in gastric cancer. Cancer 2010;116:41-9.

29 Wang S, Tie J, Wang R, et al. SOX2, a predictor of survival in gastric cancer, inhibits cell proliferation and metastasis by regulating PTEN. Cancer Lett 2015;358:210-19.

30 Muller-Immergluck MM, Schaffner W, Matthias P. Transcription factor Oct-2A contains functionally redundant activating domains and works selectively from a promoter but not from a remote enhancer position in non-lymphoid (HeLa) cells. EMBO J 1990;9:1625-34.

31 Micalizzi DS, Christensen KL, Jedlicka $\mathrm{P}$, et al. The Six1 homeoprotein induces human mammary carcinoma cells to undergo epithelial-mesenchymal transition and metastasis in mice through increasing TGF-beta signaling. J Clin Invest 2009;119:2678-90

32 Acloque $\mathrm{H}$, Adams MS, Fishwick K, et al. Epithelial-mesenchymal transitions: the importance of changing cell state in development and disease. J Clin Invest 2009;119:1438-49. 


\section{Stomach}

33 Wu W, Wong K, Chen J, et al. Directional guidance of neuronal migration in the olfactory system by the protein Slit. Nature 1999;400:331-6.

34 Brose $\mathrm{K}$, Bland $\mathrm{KS}$, Wang $\mathrm{KH}$, et al. Slit proteins bind Robo receptors and have an evolutionarily conserved role in repulsive axon guidance. Cell 1999;96:795-806.

35 Ito H, Funahashi S, Yamauchi N, et al. Identification of ROBO1 as a novel hepatocellular carcinoma antigen and a potential therapeutic and diagnostic target. Clin Cancer Res 2006;12:3257-64.

36 Grone J, Doebler O, Loddenkemper C, et al. Robo 1/Robo4: differential expression of angiogenic markers in colorectal cancer. Oncol Rep 2006;15:1437-43.

37 Bashaw GJ, Kidd T, Murray D, et al. Repulsive axon guidance: abelson and enabled play opposing roles downstream of the roundabout receptor. Cell 2000;101:703-15.

38 Guerrier S, Coutinho-Budd J, Sassa T, et al. The F-BAR domain of srGAP2 induces membrane protrusions required for neuronal migration and morphogenesis. Cell 2009;138:990-1004.
39 Chang PH, Hwang-Verslues WW, Chang YC, et al. Activation of Robo1 signaling of breast cancer cells by Slit2 from stromal fibroblast restrains tumorigenesis via blocking PI3K/Akt/beta-catenin pathway. Cancer Res 2012;72:4652-61.

40 Zhou WJ, Geng ZH, Spence JR, et al. Induction of intestinal stem cells by R-spondin 1 and Slit2 augments chemoradioprotection. Nature 2013;501:107-11.

41 Gohrig A, Detjen KM, Hilfenhaus G, et al. Axon guidance factor SLIT2 inhibits neural invasion and metastasis in pancreatic cancer. Cancer Res 2014;74:1529-40.

42 Abbas T, Dutta A. p21 in cancer: intricate networks and multiple activities. Nat Rev Cancer 2009;9:400-14.

43 Song HJ, Ming GL, Poo MM. CAMP-induced switching in turning direction of nerve growth cones. Nature 1997;388:275-9. 